\title{
Construcción de mapas de estabilidad y eficiencia de procesos de conformado de materiales metálicos policristalinos a partir de la ecuación de Garofalo
}

\author{
I. Rieiro ${ }^{(*)}$, A. Fernández $(*)$, A. Martínez ${ }^{(*)}$ y M. Carsí $i^{(*)}$
}

\begin{abstract}
Resumen Se ha construido un método sencillo y rápido para evaluar la eficiencia y condiciones de estabilidad en los procesos de conformado de materiales metálicos. Se parte de la resolución de la ecuación de Garofalo para la fluencia plástica en estado estacionario. Utilizando los parámetros de ajuste de la mencionada ecuación y de acuerdo con el significado físico de los mismos, se desarrollan las herramientas numéricas necesarias para obtener los mapas energéticos de eficiencia del tratamiento, así como las condiciones más aconsejables para el conformado del material. Además, se obtienen mapas de parámetros cuyo significado físico posee interés intrínseco, como valores de energías de activación efectivas para zonas concretas del tratamiento de los materiales y un procedimiento para obtener a partir del valor del exponente $\left\{n_{G}\right\}$ de la ecuación de Garofalo, los exponentes $\left\{n_{P L}\right\}$, característicos de las leyes potencias para intervalos reducidos de las variables de trabajo.
\end{abstract}

Palabras clave: Mapas de procesado. Conformado en caliente. Técnicas de modelización.

\section{A model for construction of efficiency and stability maps of hot working processes in polycrystalline metallic materials using the Garofalo's equation}

\begin{abstract}
Has been developed a fast and easy method for to evaluate the efficiency of the process and some of posible stabilities situations in the hot workin process for the pollicrystalines metalics materials (p.m.m.), by the obtained dates in the Garofalo's equation resolution for the steady state creep and for wide ranges of the work variables, stress, strain rate and temperature. These method use the fitting parameters obtained for the equation mentioned and of their physical meaning. Has been developed the numerical treatment from our previously developed "software" for the analysis of "creep", and we can obtained the efficiency energetic maps for the "creep" and the more generaly advises areas for the hot working. Further more has been obtained maps for parameters of great physical significance; f.e. the effective activation energy for differents areas of the materials hot working, and in addittion has been developed a method for obtained the values of $\left\{n_{P L}\right\}$ for the differents ranges of "power-law" aplication, when has been obtained the value of $\left\{n_{G}\right\}$ in the Garofalo's equation.
\end{abstract}

Key Word: Processing map. Hot forming. Modelling techniques.

\section{INTRODUCCIÓN}

El trabajo total requerido para producir una "forma" mediante la fluencia plástica en un material se puede separar en un cierto número de componentes. El trabajo que podemos denominar $W_{d}$ es

(*) Centro Nacional de Investigaciones Metalúrgicas, CENIM (CSIC), Avda. de Gregorio del Amo, 8. 28040-Madrid (España). el trabajo requerido para efectuar transformaciones homogéneas del volumen desde la sección eficaz inicial a la final, mediante deformación uniforme. Generalmente, una parte del trabajo total se gasta en trabajo redundante, $W_{r}$, también identificado como de deformación interna, y que no es otra cosa que la energía gastada en transformar el material y que no se ha invertido en cambio de forma puro. Se trata de un trabajo de transformación interna de estructura o microestructura que modifica las 
condiciones físicas del material sin producir cambio aparente de forma. En algunos casos también se suele considerar el trabajo gastado en las fricciones o resistencias con carácter disipativo de las interfases del metal y del utillaje $W_{f}$ :

$$
W_{T}=W_{d}+W_{r}+W_{f}
$$

El trabajo de acoplamiento con utillaje se suele despreciar en primera instancia. El trabajo de deformación $W_{d}$ representa la mínima energía que debe ser empleada para llevar a cabo un proceso particular de conformado, y es igual al área bajo la curva de tensión-deformación efectivas, multiplicada por el volumen:

$$
W_{d}=V \cdot \int \sigma \cdot d \varepsilon
$$

que referido a la unidad de volumen resulta más útil $w_{d}=W_{d} / V$, y en forma diferencial

$$
d w_{d}=\sigma \cdot d \varepsilon
$$

Si nos encontramos en situación de fluencia estacionaria, se podrá plantear entonces como expresión para la potencia suministrada p.e.;

$$
P_{d}=\frac{d w_{d}}{d t}=\sigma \cdot d \dot{\varepsilon}
$$

en esta situación, y a efectos de balance diferencial:

$$
d(\sigma \cdot \dot{\varepsilon})=\sigma \cdot d \dot{\varepsilon}+\dot{\varepsilon} \cdot d \sigma
$$

Con estas hipótesis tenemos:

$$
\begin{gathered}
P_{T}=\int d(\sigma \cdot \dot{\varepsilon}) \\
P_{d}=\int \sigma \cdot d \dot{\varepsilon} \\
P_{r}+P_{f}=\int \dot{\varepsilon} \cdot d \sigma
\end{gathered}
$$

Con esta descripción se puede establecer una primera constante de eficiencia del proceso:

$$
\eta_{\mathrm{I}}=\frac{P_{d}}{P_{T}}=\frac{\int_{0}^{\bar{\sigma}} \sigma \cdot d \dot{\varepsilon}}{\bar{\sigma} \overline{\dot{\varepsilon}}}
$$

Esta valoración de la eficiencia del proceso nos da una indicación del tanto por ciento de la potencia suministrada gastada en el proceso de conformado que no se ha invertido en transformaciones internas. Esta eficiencia tiene sentido si el proceso no persigue determinadas transformaciones internas, pero si el procesamiento tiene como objetivo realizar transformaciones internas que modifiquen las constantes físicas del material que son de interés no tendría sentido esta eficiencia sino $\left(1-\eta_{1}\right)$. Valores de eficiencias típicas según la definición adoptada, y aportadas por la bibliografía (1-5), en conformado se encuentran en el intervalo del 30 al $60 \%$, y en laminado por sucesivas pasadas se puede llegar a intervalos que van del 75 al $95 \%$.

A partir de este enfoque energético (termodinámico) se producen diversas aproximaciones al estudio de la estabilidad del sistema que permiten obtener parámetros de interés físico sobre el comportamiento del material en diferentes condiciones de trabajo. Así, en (1) se basan, a partir de este enfoque, en consideraciones de tipo termodinámico y abandonan el análisis microestructural que busca ecuaciones fenomenológicas potenciales para cada intervalo de trabajo posible del material. Este enfoque termodinámico no precisa de una resolución por intervalos. El método con diferente enfoque es similar a otros clásicos de control por mapas de conformados (6) en que al final por ambos métodos se pueden obtener aquellas áreas o zonas en las que la deformación es posible sin fracturas, o defectos no deseados y por ende se pueden llegar a identificar regiones óptimas en donde la deformación sea más eficiente según los objetivos buscados con la misma. A diferencia del método clásico (6) este es más general, fácil de interpretar y permite introducir en su campo de aplicación la explotación de una ecuación fenomenológica para amplios rangos de trabajo como es la ecuación de Garofalo.

Modificando ligeramente la nomenclatura anterior con la finalidad de hacer más asequibles los resultados que pretendemos:

$$
\bar{\sigma} \cdot \overline{\dot{\varepsilon}}=\int_{0}^{\bar{\varepsilon}} \sigma \cdot d \overline{\dot{\varepsilon}}+\int_{0}^{\bar{\sigma}} \overline{\dot{\varepsilon}} \cdot d \sigma
$$

y se suele presentar como $P=G+J$. La integral $G$ es la potencia gastada en deformación sin cambio en estructura, y también se denomina contenido disipado. Alternativamente, el factor $J$ es considerado el responsable de la deformación interna y se denomina co-contenido disipador.

Un primer enfoque de eficiencias lo podemos plantear como

$$
\begin{gathered}
1=\eta_{G}+\eta_{J} \\
\eta_{G}=\frac{1}{\bar{\sigma} \cdot \overline{\dot{\varepsilon}}} \cdot \int_{0}^{\bar{\varepsilon}} \sigma \cdot d \dot{\varepsilon}
\end{gathered}
$$

A una temperatura dada y para una deformación efectiva constante la partición entre $J$ y $G$ a nivel diferencial viene dada por 


$$
\begin{gathered}
\left(\frac{\partial J}{\partial G}\right)_{T, \bar{\varepsilon}}=\left(\frac{\partial \ln \bar{\sigma}}{\partial \ln \overline{\dot{\varepsilon}}}\right)_{\substack{T=\text { cte } \\
\bar{\varepsilon}=\text { cte }}}= \\
=m(\dot{\varepsilon}, T)=m(\sigma, T)
\end{gathered}
$$

siendo $m$, así definida, la sensibilidad diferencial a la velocidad del material. De esta manera se pueden determinar los valores de $m$ como función de la deformación efectiva y de la tasa de deformación, lo que de otra forma puede ser más complejo. Para poder obtener este parámetro de una ecuación fenomenológica podría parecer que esta debería depender de la deformación efectiva. Nada más lejano, ya que la manifestación de la constancia no es otra en el caso de la situación estacionaria de la fluencia que la no dependencia de la tensión eficaz respecto de la deformación eficaz. No será así en las zonas no estacionarias de la deformación plástica con independencia del fenómeno que las provoque.

Se pueden realizar dos enfoques. Uno experimental o puramente empírico sin pretender obtener conclusiones sobre la ecuación de estado o su aplicabilidad. El otro enfoque es situarnos en la hipótesis de que la ecuación fenomenológica de Garofalo lo es de estado (7) y por ende es correcta su independencia de la deformación efectiva.

Existen otros trabajos, además de los mencionados anteriormente, para el desarrollo de los mapas de conformado en la línea descrita (8-9), que no están en contradicción con la elaboración que proponemos sino que por el contrario representan métodos alternativos de aproximación al problema, según el caso, más o menos sofisticados. Creemos que nuestro método, en su fase de desarrollo actual, es eminéntemente sencillo, directo y fácil de aplicar, una vez obtenida una ecuación de Garofalo suficientemente bien ajustada para el proceso de conformado de que se trate. En este trabajo sólo queremos proponer un método para el análisis de estabilidad y eficiencia, respecto a los problemas intrínsecos del conformado, debidos a la aplicación de los esfuerzos de conformado a una velocidad y una temperatura dadas. Por otra, parte creemos que la determinación analítica de un tratamiento debe ser superior a procesos de interpolación por intervalos de trabajo.

\section{LA ECUACIÓN DE GAROFALO Y LA DEFINICIÓN DE LOS FACTORES DE EFICIENCIA DEL CONFORMADO}

Suponemos que es factible obtener las relaciones básicas de una ecuación de fluencia plástica en estado estacionario, para amplios rangos de las variables de trabajo (7):

$$
\overline{\dot{\varepsilon}}=f(\sigma, T, \bar{\varepsilon}) \quad \bar{\sigma}=f^{-1}(\overline{\dot{\varepsilon}}, T, \bar{\varepsilon})
$$

Se acepta en primera instancia como ecuación más adecuada la de Garofalo (7):

$$
\overline{\dot{\varepsilon}}=A \cdot e^{-\frac{Q}{R \cdot T}} \cdot \operatorname{senh}(\alpha \cdot \bar{\sigma})^{n}
$$

En primer lugar, es necesario estudiar y analizar el comportamiento asintótico o de condiciones de contorno de esta elección, para valorar su capacidad de aplicación.

Si $\alpha \cdot \sigma \ll$, en concreto para $\alpha \cdot \sigma<0.5$ (7) tenemos las dos aproximaciones siguientes:

$$
\begin{gathered}
\overline{\dot{\varepsilon}}=A \cdot e^{\frac{-Q}{R \cdot T}} \cdot(\alpha \cdot \sigma)^{n} \\
\bar{\sigma}=\left(\frac{1}{\alpha^{n}}\right) \cdot\left(\frac{\overline{\dot{\varepsilon}} \cdot e^{\frac{Q}{R \cdot T}}}{A}\right)^{\frac{1}{n}}
\end{gathered}
$$

Si por el contrario $\alpha \cdot \sigma \gg$, en concreto si $\alpha \cdot \sigma>$ 1.4 (7) entonces la aproximación correcta es:

$$
\begin{gathered}
\overline{\dot{\varepsilon}}=\frac{A \cdot e^{\alpha \cdot n \cdot \sigma-\frac{Q}{R \cdot T}}}{2^{n}} \\
\bar{\sigma}=\frac{1}{\alpha} \cdot \ln \left[2 \cdot\left(\frac{\overline{\dot{\varepsilon}} \cdot e^{\frac{Q}{R \cdot T}}}{A}\right)^{\frac{1}{n}}\right]
\end{gathered}
$$

Se evalúa la integral del co-contenido disipador $J$ para la ecuación de Garofalo y haciendo uso de las aproximaciones anteriores tendremos:

$$
\begin{gathered}
J=\int_{0}^{\bar{\sigma}} A \cdot e^{-\frac{Q}{R \cdot T}} \cdot \operatorname{senh}[\alpha \cdot \sigma]^{n} \cdot d \bar{\sigma} \\
J_{\alpha \sigma<<}=\frac{\overline{\dot{\varepsilon}} \cdot \bar{\sigma}}{n+1} J_{\alpha \sigma \gg>}= \\
=\left[\frac{\overline{\dot{\varepsilon}} \cdot \bar{\sigma}}{n}\right] \cdot\left[\frac{1-e^{-n \alpha \cdot \sigma}}{\alpha \cdot \sigma}\right]
\end{gathered}
$$

Por consideraciones de equilibrio se llega a la determinación de que $1 / n$ está acotado entre $[0,1]$ y por tanto $n$ lo está en el rango $[1, \infty]$. Por dicha razón se puede estimar un valor máximo de $J$, en la situación potencial o aproximación inferior y tenemos: 


$$
J_{\max (\alpha \sigma<<)}=\lim _{n \rightarrow 1} J_{\alpha \sigma}=\frac{\overline{\dot{\varepsilon}} \cdot \bar{\sigma}}{2}
$$

Por otra parte para el caso más general de evaluación para $J$ tenemos

$$
\lim _{n \rightarrow 1} J=\overline{\dot{\varepsilon}} \cdot \bar{\sigma} \cdot\left[\frac{\cosh (\alpha \sigma)-1}{(\alpha \sigma) \cdot \operatorname{senh}(\alpha \sigma)}\right]
$$

Es fácilmente demostrable que

$$
\lim _{\alpha \sigma \rightarrow 0}\left[\frac{\cosh (\alpha \sigma)-1}{(\alpha \sigma) \cdot \operatorname{sen}(\alpha \sigma)}\right]=\frac{1}{2}
$$

Además se puede probar que:

$$
\forall \alpha \sigma>0 \Rightarrow\left[\frac{\cosh (\alpha \sigma)-1}{(\alpha \sigma) \cdot \operatorname{senh}(\alpha \sigma)}\right]<\frac{1}{2}
$$

Por lo tanto es sencillo y directo que el uso de la ecuación de Garofalo, nos permite afirmar que $J / J_{\max } \leq 1 / 2$, sin necesidad de hipótesis adicionales sobre las acotaciones de $\left(n_{P L}\right)$, lo que es imprescindible desde el momento que nuestro procedimiento no va a usar los exponentes $n_{P L}$, de las Leyes Potenciales.

Con esto hemos probado que todos los desarrollos que se hacen en la bibliografía para la expresión de la ecuación tipo potencial, son consistentes con la ecuación de carácter más general que es la de Garofalo, la cual verifica en todo las mismas condiciones de contorno, y permite desarrollar las definiciones sin necesidad de hipótesis adicionales.

\section{DESARROLLO DEL MODELO TEÓRICO Y RESULTADOS}

La introducción y el uso de los coeficientes que vamos a usar precisa de algunas explicaciones sobre su base física.

La función de Liapunov de un sistema es una cantidad creada "ad-hoc" para el mismo y asociado al criterio de estabilidad de Liapunov para el sistema (12). Es una función arbitraria, y en algunos casos más de una, que relaciona los cambios en la energía total del sistema. El criterio de estabilidad de Liapunov requiere que el sistema minimice su energía total continuamente. Una función de Liapunov es:

$$
V_{1}=\eta \cdot(\ln (\dot{\varepsilon})) \quad \eta=\frac{J}{J_{\max }}=\frac{2 \cdot m}{m+1}
$$

dando lugar a la condición $1^{\text {a }}$ :

$$
\frac{\delta \eta}{\delta \ln (\dot{\varepsilon})}<0
$$

condición que define la región estable. Esta condición asegura que el sistema se aproxima a la condición de estado estacionario en la que experimenta un estado de mínima energía y un máximo rendimiento sin fractura (10-11). Es directo que la anterior condición se puede sustituir por:

$$
\frac{\delta m}{\delta \ln (\dot{\varepsilon})}<0
$$

Por otra parte, no basta con este factor. Además, es necesaria la obtención de un parámetro de control que evalúe la influencia de la temperatura sobre el material y su capacidad de transformación. La potencia total disipada se representa en términos de la tasa de entropía aplicada al sistema $y$ de $T$ :

$$
P_{T}=\bar{\sigma} \cdot \dot{\bar{\varepsilon}}=\dot{S}_{\text {input }} \cdot T
$$

Como la tasa de entropía producida por el sistema es:

$$
\dot{S}_{\text {output }}=\left[\frac{\delta P}{\delta T}\right]_{\dot{s}}=\frac{P_{T}}{T^{2}} \cdot \frac{\delta \ln (\bar{\sigma})}{\delta(1 / T)}=\frac{P_{T}}{T} \cdot s
$$

resulta:

$$
s=\frac{\dot{S}_{\text {output }}}{\dot{S}_{\text {input }}}=\frac{1}{T} \cdot \frac{\partial \ln (\sigma)}{\partial(1 / T)}
$$

y, de acuerdo con el $2^{\circ}$ principio de la termodinámica, $s$ debe ser mayor que 1 para la fluencia estable del material y esto se traduce en (10):

$$
\frac{\delta s}{\delta(\ln (\dot{\varepsilon}))}<0
$$

descansando en el fondo de haber construido una $2^{\mathrm{a}}$ función de Liapunov:

$$
V_{2}=s \cdot(\ln (\dot{\varepsilon}))
$$

Por lo tanto, las dos condiciones que asumimos de forma conjunta para la condición de estabilidad del trabajo de conformado en caliente en fluencia plástica en estado estacionario son: 


$$
\frac{\delta m}{\delta \ln (\dot{\varepsilon})}<0 \quad \frac{\delta s}{\delta(\ln (\dot{\varepsilon}))}<0
$$

Las áreas sobre las regiones de las variables de trabajo que verifican estas dos condiciones simultáneas nos aportan el mapa de la región de estabilidad buscada.

A partir de los resultados anteriores debemos adoptar para $J$ :

$$
J=\frac{A \cdot e^{-\frac{Q}{R T}}}{\alpha} \int_{0}^{\alpha \cdot \sigma} \operatorname{senh}(x)^{n} \cdot d x
$$

De donde la eficiencia que hemos definido anteriormente:

$$
\eta_{J}=\frac{J}{\sigma \cdot \dot{\varepsilon}}=\left\{\frac{\int_{0}^{\alpha \cdot \sigma} \operatorname{senh}(x)^{n} \cdot d x}{(\alpha \cdot \sigma) \cdot(\operatorname{senh}(\alpha \cdot \sigma))^{n}}\right\}
$$

$y$, por tanto, trivialmente

$$
\eta_{G}=1-\eta_{J}
$$

Para procesos integrales resulta oportuno definir:

$$
\frac{J}{G}=\frac{\varphi_{1}(\alpha, n, \sigma)}{\varphi_{2}(\alpha, n, \sigma)-\varphi_{1}(\alpha, n, \sigma)}
$$

siendo

$$
\begin{gathered}
\varphi_{1}(\alpha, n, \sigma)=\int_{0}^{\alpha \cdot \sigma} \operatorname{senh}(x)^{n} \cdot d x \\
\varphi_{2}(\alpha, n, \sigma)=(\alpha \cdot \sigma) \cdot(\operatorname{senh}(\alpha \cdot \sigma))^{n}
\end{gathered}
$$

Resulta necesario definir la eficiencia a partir de otras variables de uso en los ensayos de fluencia, lo cual va a permitir observar el comportamiento de la eficiencia a partir de velocidades de deformación y temperaturas:

$$
\begin{aligned}
& \eta_{J}=\left(\frac{\dot{\varepsilon} \cdot e^{\frac{Q}{R \cdot T}}}{A}\right)^{\frac{1}{n}} \cdot\left(\frac{1}{\frac{1}{\alpha} \cdot \operatorname{arcsenh}\left[\left(\frac{\dot{\varepsilon} \cdot e^{\frac{Q}{R \cdot T}}}{A}\right)^{\frac{1}{n}}\right]}\right) \\
& \cdot \int_{0}^{\frac{1}{\alpha} \operatorname{arcsconh}}\left[\left(\frac{\dot{\varepsilon} \cdot \frac{Q}{R T}}{A}\right)^{\frac{1}{n}}\right] \operatorname{senh}(\alpha \cdot \sigma) \cdot d \sigma
\end{aligned}
$$

Algunas sencillas transformaciones algebraicas permiten otra expresión útil:

$$
\begin{aligned}
& \eta_{J}= \frac{1}{n \cdot \dot{\varepsilon} \cdot \operatorname{arcsenh}\left[\left(\frac{\dot{\varepsilon} \cdot e^{\frac{Q}{R T}}}{A}\right)^{\frac{1}{n}}\right]} . \\
& \cdot \int_{0}^{\dot{\varepsilon}} \frac{1}{\sqrt{1+\left(\frac{\dot{\varepsilon} \cdot e^{\frac{Q}{R T}}}{A}\right)^{\frac{-2}{n}}} \cdot d \dot{\varepsilon}}
\end{aligned}
$$

Debido a que hemos acotado $J_{\max }$, y podemos sostener que $J_{\max }<(\varepsilon \cdot \sigma) / 2$, se puede definir la eficiencia, según los patrones tradicionales en la bibliografía, como:

$$
\eta_{J(b)}=2 \cdot \eta_{J}
$$

Esta eficiencia $\eta=J / J_{\max }$ únicamente aporta un referente respecto al comportamiento de un material ideal $\operatorname{con} n=1$.

En el caso en el que la ecuación de fluencia siga una ley potencial, se obtiene, como es sabido

$$
J=\left(\frac{m}{m+1}\right) \cdot \sigma \cdot \dot{\varepsilon}
$$

y además

$$
G=\left(\frac{1}{m+1}\right) \cdot \sigma \cdot \dot{\varepsilon}
$$

de donde

$$
\frac{J}{G}=m
$$

Ahora bien, estas relaciones no se pueden ni generalizar ni extrapolar al caso de otras relaciones funcionales entre velocidad de deformación y tensión, como es el caso de la ecuación de Garofalo. Tampoco se puede identificar el valor de $m=1 / n$, siendo $n$ el exponente de la ley potencial con el valor de la sensibilidad diferencial $m$. La sensibilidad diferencial definida como

$$
m=\left[\frac{\partial \ln (\sigma)}{\partial \ln (\dot{\varepsilon})}\right]_{T, \varepsilon}
$$

es la expresión de carácter general a aplicar, ya que, por ejemplo, en el caso de usar la ecuación de Garofalo tendríamos la siguiente expresión:

$$
m=\left(\frac{1}{n}\right) \cdot \frac{\tanh (\alpha \cdot \sigma)}{\alpha \cdot \sigma}
$$


y es directo que:

$$
\lim _{\alpha \cdot \sigma \rightarrow 0} m \Rightarrow \frac{1}{n} \quad \lim _{\alpha \cdot \sigma} m \Rightarrow 0
$$

Luego, podemos establecer que para $(n)$, exponente en la ecuación de Garofalo:

$$
0<m<\frac{1}{n_{G}}
$$

Ésta es la relación que debe cumplir el exponente de la ecuación de Garofalo y, además de unos principios generales de acotación, podemos obtener la acotación del valor de " $n$ " exponente en la ecuación de Garofalo.

Es conveniente plantear las expresiones alternativas de la sensibilidad diferencial, a partir de una variable que resulta práctica (obsérvese que no es otra cosa que el factor de Zener normalizado a la constante de entropía de la ecuación de Garofalo).

$$
\theta(\dot{\varepsilon}, T)=\frac{\dot{\varepsilon} \cdot e^{\frac{Q}{R: T}}}{A}
$$

a partir de esta variable auxiliar obtenemos la siguiente expresión:

$$
m=\left(\frac{1}{n}\right) \cdot \frac{1}{\operatorname{arcsenh}\left(\theta^{\frac{1}{n}}\right)} \cdot \frac{\theta^{\frac{1}{n}}}{\sqrt{1+\theta^{\frac{2}{n}}}}
$$

con lo que tenemos una relación entre la sensibilidad diferencial y el factor de Zener. Podemos además definir un factor de entropía diferencial del sistema necesario para verificar la estabilidad del proceso a partir de valoraciones del balance energético del proceso [10], [2-5] como:

$$
\begin{gathered}
s=\frac{1}{T} \cdot \frac{\partial \ln (\sigma)}{\left.\partial\left(\frac{1}{T}\right)\right]_{\varepsilon, \dot{\varepsilon}}}= \\
=\left(\frac{Q}{n \cdot R \cdot T}\right) \cdot \frac{\theta^{\frac{1}{n}}}{\operatorname{arcsenh}\left(\theta^{\frac{1}{n}}\right) \cdot \sqrt{1+\theta^{\frac{2}{n}}}}= \\
=\left(\frac{Q}{n \cdot R \cdot T}\right) \cdot\left(\frac{\operatorname{tangh}(\alpha \cdot \sigma)}{\alpha \cdot \sigma}\right)
\end{gathered}
$$

A los efectos de posteriores estudios sobre la estabilidad de las situaciones de tratamiento y conformado, nos interesa definir dos variaciones diferenciales que tendrán en cuenta los principios de estabilidad de Liapunov, que son [10-11], [1], [4]:

$$
\begin{gathered}
\frac{\partial m}{\partial \ln (\dot{\varepsilon})}=\frac{1}{n^{2}} . \\
{\left[\frac{\theta^{\frac{1}{n}} \cdot \operatorname{arcsenh}\left(\theta^{\frac{1}{n}}\right)-\theta^{\frac{2}{n}} \cdot \sqrt{1+\theta^{\frac{2}{n}}}}{\left[\operatorname{arcsenh}\left(\theta^{\frac{1}{n}}\right)\right]^{2} \cdot\left(1+\theta^{\frac{2}{n}}\right)^{\left(\frac{3}{2}\right)}}\right]}
\end{gathered}
$$

De la misma forma definimos la variación diferencial del coeficiente ligado a la entropía:

$$
\begin{gathered}
\frac{\partial s}{\partial \ln (\dot{\varepsilon})}=\left(\frac{1}{n^{2}}\right) \cdot\left(\frac{Q}{R \cdot T}\right) \cdot \\
{\left[\frac{\theta^{\frac{1}{n}} \cdot \operatorname{arcsenh}\left(\theta^{\frac{1}{n}}\right)-\theta^{\frac{2}{n}} \cdot \sqrt{1+\theta^{\frac{2}{n}}}}{\left(1+\theta^{\frac{2}{n}}\right)^{\frac{3}{2}} \cdot\left[\operatorname{arcsenh}\left(\theta^{1}\right)\right]^{2}}\right]}
\end{gathered}
$$

De la formulación anterior resulta sencilla la obtención de unos coeficientes de interés para el estudio del comportamiento físico del conformado del material, a partir de la Ecuación de Garofalo.

Teniendo en cuenta que cuando se considera una expresión potencial para la ecuación de estado en fluencia se llega a establecer una relación $n=1 / m$, podemos definir un coeficiente $n_{\text {efectivo }}$ que sirve para restituir el valor del coeficiente $n_{\mathrm{PL}}$, según las diferentes zonas de trabajo (7):

$$
n_{\text {efectivo }}=\frac{1}{\left(\frac{\partial \ln (\sigma)}{\partial \ln (\dot{\varepsilon})}\right)_{T, \varepsilon}}
$$

Igualmente, definimos un volumen de activación efectivo como (13):

$$
V_{\text {efectivo }}=R \cdot T \cdot\left(\frac{\partial \ln (\varepsilon \cdot)}{\partial \sigma}\right)_{T}
$$

y una energía de activación efectiva [7]:

$$
Q_{\text {efectivo }}=-R \cdot\left(\frac{\partial \ln (\dot{\varepsilon})}{\partial\left(\frac{1}{T}\right)}\right)
$$




\section{CONCLUSIONES}

Las formulas de [33], [34], [35], [36] y [37] para los cálculos de la eficiencia [47] y [48] para los análisis de la estabilidad, y [49], [50] y [51] para el estudio de parámetros físicos de interés en el procesado, resumen un modelo compacto sencillo y directo cuya aplicación permite:

- Obtener mapas de eficiencia del rendimiento de los procesos de fluencia

- Obtener mapas de la sensibilidad diferencial con diferentes variables

- Obtener mapas de zonas de estabilidad e inestabilidad del comportamiento de los materiales en los procesos de fluencia

- Obtener representaciones de valores de $n_{\text {efectivo, }}$, $Q_{\text {efectivo }}, V_{\text {efectivo }}$

- Estudiar los valores de los exponentes de las leyes potenciales que habría sido necesario utilizar para describir con ellas el mismo fenómeno y proceso.

- Valorar de forma rápidamente el rendimiento energético del material en diferentes regiones de trabajo.

\section{REFERENCIAS}

(1) Prasad, Y.V.R.K., Gegel, H.L., Davaivelu, S.M., Malas, J.C., Morga, J.T., Lark, K.A. y Barker, D.R. Metall. Trans., October, 1994, p. 1883.
(2) RAJ, R. Metall. Trans. A, 12 A, 1981, p. 1089.

(3) Gegel, H.L., Nodiv, S. y RaJ, R. Scr. Met., 14, 1980, p. 235

(4) Gegel, H.L. Proceeding of the AUTO FACT-1987, Conference Detroit, MJ., 1987.

(5) RaJ, R. y Ashby, M.F. Metall. Trans., 3, 1972, p. 1937.

(6) Frost, H.J. y Ashby, M.F. "Deformation Mechanism Maps”, Pergamon Press, Oxford, 1982.

(7) RiEIRo, I. "Estudio y resolución de la ecuación fenomenológica de Garofalo para la fluencia plástica en estado estacionario de materiales metálicos policristalinos. Determinación de su capacidad de aplicación y base Física". Tesis Doctoral. Universidad Complutense, Junio, 1997.

(8) Al OMAR, A. "Caracterización dinámica de dos aceros microaleados de medio carbono mediante ensayos de compresión a alta temperatura. Aplicación de mapas de procesado", Tesis Doctoral, Universidad Politécnica de Cataluña, Febrero, 1996.

(9) Gegel, H.L. y Malas, J.C. $2^{\text {nd }}$ Int. Conf on the Technology of Plasticity, Stuttgart, FRG. 1987., p. 439.

(10) Gegel, H.L. y Malas, J.C. $2^{\text {nd }}$ Int. Conf on the Technology of Plasticity, Stuttgart, FRG. 1987., p. 417.

(11) SRinivasan, N. y Prasad, Y.V.R.K. Metall. Mat. Trans. A", 25 A, 1994, p. 2275.

(12) Schultz, D.L. y Melsa, J.L. "State Functions and Lineal Control Systems”, McGraw Hill, 1967.

(13) Sherby, O.D., Robbins, J.L. y Goldberg, A. J. App. Physic, 41, 1970, 3961-1968. 